\title{
ACOLHIMENTO EM UNIDADES DE PRONTO ATENDIMENTO: PERCEPÇÃO DE IDOSOS E SEUS FAMILIARES
}

\section{USER EMBRACEMENT IN EMERGENCY CARE UNITS: PERCEPTION OF ELDERLY AND THEIR FAMILIARS}

\section{ACOGIDA EN UNIDADES DE SERVICIOS DE EMERGENCIA: PERCEPCIÓN DE LOS ANCIANOS Y SUS FAMILIARES}

Giovana Aparecida de Souza Scolari ${ }^{1}$, Leidyani Karina Rissardo², Vanessa Denardi Antoniassi Baldissera ${ }^{3}$, Celmira Lange ${ }^{4}$, Maria Aparecida Salci5 ${ }^{5}$ Ligia Carreira ${ }^{5}$.

\section{RESUMO}

Objetivo: Compreender as práticas de acolhimento em Unidades de Pronto Atendimento na perspectiva de idosos e familiares à luz da Política Nacional de Humanização. Métodos: Estudo qualitativo realizado junto a vinte idosos e cinco familiares, que foram atendidos em duas Unidades de Pronto Atendimento. Os dados foram coletados, por meio de entrevistas semiestruturadas, e a análise conduzida pelas etapas analíticas da Grounded Theory. Resultados: Evidenciou-se que o acolhimento é percebido entre as práticas de receptividade, ao entrar no serviço e na escuta qualificada. Entretanto, também foram constatadas algumas dificuldades, como a demora pelo atendimento e aspectos referentes a ambiência e infraestrutura que prejudicam a acomodação adequada dos usuários. Conclusão: Apesar dos idosos sentirem-se satisfeitos, em decorrência de as necessidades de saúde serem supridas, há urgência na reestruturação e na organização dos serviços, capacitação profissional e estratégias de atendimento que proporcionem a esta população uma assistência com responsabilidade.

Descritores: Acolhimento; Humanização da Assistência; Enfermagem em Emergência; Idoso; Enfermagem Geriátrica.

\section{ABSTRACT}

Objective: to understand the welcoming practices in Emergency Care Units from the perspective of the elderly and their family members in the light of the National Humanization Policy. Methods: Qualitative study carried out with twenty elderly people and five family members, who were assisted at two Emergency Care Units. Data were collected by means of semi-structured interviews, and the analysis was conducted using the analytical steps of Grounded Theory. Results: It was evidenced that welcoming is perceived as one of the user embracement practices when entering the service and qualified listening. However, some difficulties were also observed, such as delays in service and aspects related to the ambience and infrastructure that hinder the adequate accommodation of users. Conclusion: Although the elderly feel satisfied by their health needs being met, there is an urgent need for restructuring and organizing services, promoting professional training and creating care strategies that provide this population with responsible care.

Descriptors: User Embracement; Humanization of Assistance; Emergency Nursing; Aged; Geriatric Nursing.

\section{RESUMEN}

Objetivo: comprender las prácticas de acogida en las Unidades de Atención de Emergencia desde la perspectiva de los ancianos y los miembros de la familia a la luz de la Política Nacional de Humanización. Métodos: estudio cualitativo realizado con veinte personas mayores y cinco familiares, atendidos en dos Unidades de Atención de Emergencia. Los datos se recopilaron a través de entrevistas semiestructuradas, y el análisis se realizó utilizando los pasos analíticos de Grounded Theory. Resultados: se evidenció que la acogida se percibe entre las prácticas receptivas al ingresar al servicio y en la escucha calificada. Sin embargo, también se observaron algunas dificultades, como la demora en el servicio y aspectos relacionados con el ambiente y la infraestructura que dificultan el alojamiento adecuado de los usuarios. Conclusión: aunque los ancianos se sienten satisfechos debido a que se cubren sus necesidades de salud, existe una necesidad urgente de reestructurar y organizar servicios, capacitación profesional y estrategias de atención que brinden a esta población una atención responsable.

Descriptores: Acogimiento; Humanización de la Atención; Enfermería de Urgencia; Anciano; Enfermería Geríatrica.

${ }^{1}$ Doutoranda em Enfermagem pelo Programa de Pós-Graduação em Enfermagem da Universidade Estadual de Maringá, Maringá-PR. ${ }^{2}$ Doutora em Enfermagem, Docente do Departamento de Medicina, Unicesumar. ${ }^{3}$ Doutora em Ciências, Docente do Departamento de Enfermagem da Universidade Estadual de Maringá. ${ }^{4}$ Doutora em Enfermagem, Docente da Universidade Federal de Pelotas. ${ }^{5}$ Doutora em Enfermagem, Docente do Departamento de Enfermagem da Universidade Estadual de Maringá.

Como citar este artigo:

Scolari GAS, Rissardo LK, Baldissera VDA, et al. Acolhimento em unidades de Pronto Atendimento: experiências relatadas pelos Idosos e seus familiares. Revista de Enfermagem do Centro Oeste Mineiro. 2020;10:e3726. [Access ]; Available in: DOI: http://doi.org/10.19175/recom.v10i0.3726 


\section{INTRODUÇÃO}

O envelhecimento populacional tem progredido com outros fatores, como as condições crônicas que acometem grande parte da população idosa e está, intensamente, associada às fragilidades dessa faixa etária. As Doenças Crônicas Não Transmissíveis (DCNT) representam importante desafio para a saúde pública, provocando incapacidades que afetam a qualidade de vida dos indivíduos e a economia dos países ${ }^{(1)}$. Esse fenômeno revela novas demandas para o sistema de saúde, e atrelado aos desafios existentes na rede de atenção ao idoso traz repercussões para os serviços de urgência e emergência ${ }^{(2)}$, principalmente, às Unidades de Pronto Atendimento (UPAs).

As UPAs foram implantadas, em 2009, a partir da reestruturação do atendimento às urgências e emergências consolidada pela Política Nacional de Atenção às Urgências (PNAU), configurando-se como serviços de saúde não hospitalares que visam a estabelecer a relação entre as Unidades Básicas de Saúde (UBS) e a Estratégia de Saúde da Família (ESF) com a rede hospitalar. Como atribuições desempenham papel relevante no atendimento à saúde de média complexidade, possibilitando acesso aos usuários idosos nesse nível de atenção ${ }^{(3)}$.

A estruturação da PNAU ocorreu no mesmo período de elaboração da Política Nacional de Humanização (PNH), o que constitui considerável passo para a qualificação dos serviços de emergência, especialmente no que compreende o acolhimento, a humanização e a organização do sistema de urgência e emergência, estabelecido pela classificação de risco, em que o usuário é atendido de acordo com seu estado de saúde ${ }^{(4-5)}$.

$\mathrm{O}$ ato de acolher excede a perspectiva do acesso ao serviço de saúde, perpassa uma ação de porta de entrada e recepção do usuário(4-6). Portanto, o acolhimento é compreendido como um processo contínuo que exige dos profissionais de saúde sensibilidade, conhecimento e responsabilização ativa pela condição de saúde do idoso ${ }^{(4-5)}$, com associação das três esferas constitutivas na realização dessa prática: postura, atitude e tecnologia do cuidado; mecanismo de ampliação e facilitação do acesso; e, dispositivo de (re)organização do processo de trabalho em equipe ${ }^{(5)}$.

A literatura aponta estudo que versa o acolhimento ao idoso na Atenção Primária à Saúde $(A P S)^{(7)}$ e até em serviços de emergência hospitalares ${ }^{(4)}$, mas pesquisas com esse público nas UPAs são escassas, havendo necessidade de compreensão acerca do acolhimento na assistência ofertada a esse grupo populacional e a importância dessas Unidades na organização do cuidado, a partir da percepção dos próprios usuários idosos e de seus familiares.

Nesse ínterim, surgiu o questionamento: Qual a percepção de idosos e familiares sobre as práticas de acolhimento em UPAs? Acredita-se que a resposta possibilitará o enfoque ao desenvolvimento das ações do acolhimento, compreendendo em que situações e o modo como estão sendo desenvolvidas, o que pode contribuir no aprimoramento dessa atividade por gestores e profissionais, a fim de propiciar melhorias na qualidade dos serviços ofertados. Assim, o presente estudo tem como objetivo compreender as práticas de acolhimento em UPAs na perspectiva de idosos e familiares à luz da Política Nacional de Humanização.

\section{MÉTODO}

Estudo qualitativo que teve como referencial teórico a PNH e metodológico as técnicas analíticas da Grounded Theory. Realizado em duas UPAs de um município de médio porte da região Sul do país, responsáveis pelo atendimento intermediário de caráter emergencial do município.

Para a seleção dos participantes foi realizado um sorteio entre os indivíduos com idade igual ou superior a 60 anos, que foram atendidos nas UPAs, no mês de novembro de 2015. Esse período corresponde a um mês antes do início da coleta de dados, que ocorreu entre dezembro de 2015 a abril de 2016, junto a vinte idosos e cinco familiares, no intuito de propiciar recordação do processo de acolhimento durante a assistência à Unidade.

O primeiro contato com os participantes foi via telefone, por intermédio de uma funcionária da UPA, à qual foram feitos convites prévios para participar do estudo e agendados horários com os mesmos.

No encontro presencial, foi verificada a condição cognitiva do idoso, de acordo com o teste Miniexame do Estado Mental (MEEM), levando em consideração o nível de escolaridade ${ }^{(8)}$, sendo 20 pontos para analfabetos, 25 para indivíduos com até quatro anos de estudos, 26,5 para àqueles que estudaram de cinco a oito anos, 28 para pessoas com nove a 11 anos de escolaridade e 29 para quem tem escolaridade superior a $11 \operatorname{anos}^{(8)}$. E ainda, que 
estivesse em condição clínica para participar do estudo. Para os idosos que não apresentaram condição cognitiva preservada, foram convidados, seus familiares, em que os seguintes critérios foram respeitados: também possuir capacidade cognitiva avaliada pelo MEEM e ser cuidador principal do idoso.

Os participantes foram orientados sobre a pesquisa e seus objetivos, o caráter voluntário e a necessidade de gravação das falas. As entrevistas tiveram duração média de 26 minutos, sendo realizada a maioria (23) no domicílio do idoso, utilizando um roteiro de coleta de dados que teve adequação de seu conteúdo por especialistas da área ${ }^{(9)}$. Este foi constituído por três partes: características sociodemográficas dos idosos e sua família; características do atendimento; e, questões acerca do acolhimento durante 0 atendimento na UPA, que foram elaboradas em conformidade à $\mathrm{PNH}^{(5)}$ : "Para o Sr.(a), o que é acolhimento?", "Como foi o seu acolhimento na UPA X?". As entrevistas foram realizadas por uma enfermeira, mestranda, com experiência em pesquisa qualitativa e integrante de um grupo de estudo e pesquisa sobre envelhecimento vinculado a uma universidade paranaense.

A coleta e a análise dos dados ocorreram em concomitância, após cada entrevista era realizado a transcrição das falas e sua análise, sendo essencial para guiar a seleção dos próximos participantes. Durante todo o percurso desta etapa, foi respeitado os critérios de amostragem e saturação teórica, momento em que não se evidenciou o surgimento de códigos novos ${ }^{(10)}$.

Ao desenvolver o método comparativo constante, como propõem Strauss e Corbin ${ }^{(10)}$ observou-se que a condição clínica do participante influenciava no entendimento sobre o acolhimento realizado nas UPAs, sendo necessário realizar o estudo com idosos classificados por todas as cores, segundo o Protocolo de Manchester utilizado nas Unidades ${ }^{(11)}$, Azul, Verde, Amarela e Vermelha, a fim da melhor compreensão do objeto de estudo.

Assim, as entrevistas foram realizadas, inicialmente, com oito idosos classificados pela coloração Verde; seguido, de sete idosos, classificados pela cor Amarela; cinco classificados em Azul; e, cinco familiares de idosos com declínio cognitivo e situações clínicas instáveis e de maior gravidade, classificados com a cor Vermelha.

Para a análise dos dados foram adotadas as técnicas analíticas abertas e axial da Grounded Theory, por possibilitar uma sistematização técnica para os procedimentos de coleta e análise que enfatiza a importância em conhecer, entender e interpretar a natureza dos eventos e situações $^{(10)}$. Na codificação aberta, os dados foram analisados linha por linha a fim de identificar cada incidente, gerando 35 códigos que, depois de agrupados promoveram a elaboração dos conceitos. Na codificação axial, os dados foram reagrupados com a finalidade de obter uma explicação mais clara e completa sobre o fenômeno em estudo, associando categorias às subcategorias, por meio de um processo analítico sistematizado de comparação e conexão dos $\operatorname{dados}^{(10)}$.

A utilização dessas etapas permitiu, durante a codificação aberta identificar como os idosos compreendiam as práticas de acolhimento nas UPAs e, por meio da codificação axial, a reunião dos códigos em dois eixos, correspondente as duas categorias: 'Satisfação com o acolhimento das UPAs' e 'Dificuldades encontradas no processo de acolhimento nas UPAs'.

$O$ estudo foi realizado em conformidade com as exigências das Resoluções 466/2012 e 510/2016, com aprovação do projeto pelo Comitê Permanente de Ética em Pesquisa com Seres Humanos da Universidade Estadual de Maringá, sob o CAAE: 51328915.3.0000.0104 e Parecer no 1. 375.173. Todos os participantes assinaram o Termo de Consentimento Livre e Esclarecido. O anonimato foi garantido e os discursos identificados com a letra "I" para os Idosos e "F" para os Familiares, seguido do número da entrevista e a classificação de risco obtida.

\section{RESULTADOS E DISCUSSÃO}

Dos 20 idosos participantes da pesquisa, a idade variou entre 60 e 85 anos, com média de 69 anos. Houve predominância do sexo feminino (14) e o estado civil da maioria era casado (13), 17 tinham o nível de escolaridade de até quatro anos de estudo. As principais queixas de saúde que os levaram a procurar as UPAs estavam relacionadas às doenças do sistema osteomuscular e do tecido conjuntivo (9).

Quanto aos cinco familiares, três eram filhas dos idosos, uma esposa e uma ex-nora, a idade esteve entre 40 e 78 anos, com média de 61 anos. Todos eram do sexo feminino, quatro desenvolviam atividade laboral de cuidado ao idoso e afazeres domésticos do lar, três possuíam até oito anos de estudo. A principal queixa que motivaram os familiares a levarem os idosos nas 
UPAs estava relacionada as Doenças Cardiovasculares.

\section{Satisfação com o acolhimento das UPAs}

Nessa categoria são apresentadas as satisfações com o acolhimento nas Unidades estudadas, a qual, na perspectiva dos participantes, esteve relacionada à organização do serviço, à classificação de risco, às tecnologias leves de escuta qualificada e vínculo, que foram apontadas positivamente na receptividade ao serviço e no modo de atender dos profissionais, que está entre as diretrizes da $\mathrm{PNH}$.

Entre os aspectos do acolhimento apontados como satisfatório pelos idosos e familiares, está a organização do serviço, especialmente, no que se refere ao Acolhimento Com Classificação de Risco (ACCR): “(...) acho certa esta classificação que eles fazem na UPA, porque quando você está com uma coisa que pode esperar, você pode até ir no posto" (I12-Amarelo).

Segundo os participantes da pesquisa, ao chegar à UPA, o usuário é classificado por quatro cores distintas e de acordo com sua condição clínica, define-se o desfecho do atendimento: “(...) a enfermeira me atendeu, viu que minha pressão estava um pouco alterada, aí ela me deu a ficha amarela, porque quando você está com emergência, o médico atende primeiro" (I12Amarelo). "Quando chego lá, sou bem recebido. Se tem gente na minha frente, aguardo um pouco e logo sou chamado para fazer a triagem. Se for caso de emergência, da triagem mesmo já me passa para dentro. Quando não é emergência, faço a triagem, volto para a recepção, sento na cadeira, fico aguardando e o médico me chama" (I7-Verde).

O ACCR é um importante dispositivo implantado nos serviços de emergência a partir da $\mathrm{PNH}^{(5)}$. Essa ferramenta tem como função primordial integrar o acolhimento, humanizando o atendimento, por meio da promoção de práticas e ações de saúde voltadas à institucionalização do cuidado aos usuários nos serviços de urgência e emergência com qualidade, por meio de ordem de fluxo, priorizando os casos mais graves ${ }^{(12)}$. O método de acolhimento utilizado, nas UPAs estudadas, faz com que o indivíduo seja avaliado por um enfermeiro, imediatamente, ao chegar na Unidade e, a partir da sua condição clínica é definida a classificação de risco correspondente, o que determina se deve obter atendimento médico imediato ou se poderá aguardar(11).
Outro aspecto referido como satisfatório sobre o acolhimento na UPA, diz respeito à necessidade de acompanhamento laboratorial para a estabilidade de enfermidades, como nos casos dos idosos com DCNT: "Quando cheguei na UPA, disse na recepção que tinha ido para fazer o exame e mostrei o papel. A moça já me chamou. Fui bem atendido. Nem cheguei a sentar para esperar. Depois que colheu o sangue, ela falou assim: espera um pouquinho que já dou o resultado" (I19-Azul). A disponibilização dos serviços oferecidos nas UPAs, chamam a atenção dos usuários por causa da ampla possibilidade de acesso a exames e resolutividade no atendimento $^{(13)}$.

Em todo o processo de atendimento, seja na classificação de risco, na sala de medicação ou na consulta médica, foi notória a satisfação dos idosos e seus familiares ao discorrerem sobre a empatia dos profissionais na assistência prestada, referendada por eles como sinônimo de acolhimento: "O acolhimento é a maneira deles tratarem a gente, acolher com carinho e dedicação. Quando estive na UPA acompanhando minha mãe, toda hora estavam perguntando como ela estava. Isso dá mais força para gente. (...) O acolhimento foi ótimo!" (F23-Vermelho).

$\mathrm{O}$ ato de acolher não se limita apenas a uma recepção cordial, mas inclui a escuta ativa do usuário, vínculo, resolutividade e desempenho profissional. A escuta qualificada é elemento crucial ao processo de acolhimento, pois visa promover uma resposta positiva e responsável pela resolução de sua necessidade, por meio de práticas interventivas, que é o produto final do trabalho em saúde ${ }^{(4-6)}$.

Apesar de os serviços de emergência serem permeados por rotina acelerada e propícios ao desenvolvimento do tecnicismo, por parte dos profissionais, é notória a humanização na assistência prestada, valorizando a família como cuidadora, respeitando os aspectos legais, proporcionando-lhes suporte diante da situação vivida, com responsabilidade e dedicação. A empatia faz parte do processo de humanização, sendo de extrema importância para a valorização da assistência e a qualidade do cuidado, os quais são reconhecidos pelos participantes do estudo como os principais aspectos que geram satisfação com o serviço.

Associada às práticas de acolhimento intermediadas pelos profissionais, foi mencionado pelos participantes o direito do idoso de ter acompanhante, sendo elogiada a maneira com 
que os acolhem, inclusive pelo oferecimento de alimentação durante a estadia: "Os funcionários da UPA até avisaram que, se quisesse ficar alguém como acompanhante, devido minha idade, poderia" (I10-Amarelo). "Quando precisei ir a UPA, sempre estava tudo organizado (...). Ainda, eles me davam alimentação, porque eu ficava acompanhando minha mãe" (F23-Vermelho).

Tais fatores são primordiais quando se trata do público idoso, independente da condição clínica, necessitam de atenção qualificada, em razão das limitações funcionais e/ou cognitiva ou, ainda, por estarem em um ambiente totalmente estranho. Nesse contexto, uma das práticas que podem facilitar o cuidado desses indivíduos é o acompanhamento por alguém de sua preferência. Fato mencionado, positivamente, pelos idosos deste estudo, os quais receberam essa informação, desde a entrada no serviço. Ressaltase que também está entre as diretrizes da $\mathrm{PNH}, \mathrm{O}$ direito do idoso ter acompanhante durante sua permanência nos serviços de saúde ${ }^{(5)}$.

Além de possibilitar o acompanhamento, nesta pesquisa foi explicitado outro atributo importante do acolhimento, o fornecimento de alimentação para o acompanhante. Sabe-se que o suprimento das necessidades fisiológicas está entre as estratégias para funcionamento adequado da UPA ${ }^{(3)}$. E muito além de suprir as necessidades humanas básicas do usuário, dentre as funções do ato de se alimentar, tem-se a função hedônica, pelo prazer de comer, e conviver, uma vez que fazer as refeições faz parte da cultura do indivíduo ${ }^{(15)}$, e isso está intimamente relacionado à prática de humanização.

Entre os aspectos satisfatórios da organização do processo de trabalho das Unidades, tem-se o seguimento da assistência e alcance das expectativas do indivíduo, sendo esta considerada fundamental ao atendimento dos idosos nas UPAs: "Primeiro passei pela recepção, depois pelo médico e para a sala de medicação. Então, deram encaminhamento para o posto. Tinha bastante gente, mas me chamaram logo. Eles tratam a gente muito bem" (I18-Azul).

Pela dinâmica de trabalho relatada pôde ser percebido, entre as práticas de acolhimento, a continuidade do cuidado expressado pelo encaminhamento a outro serviço. Quando o indivíduo procura a Unidade, por condições sensíveis à atenção primária, geralmente o médico realiza o atendimento necessário, depois o encaminha para a APS para que seja assistido com longitudinalidade ${ }^{(15)}$, característica preponderante dos idosos que possuem alta frequência de agudizações de suas condições crônicas. Além de que, é imprescindível que a atenção primária exerça suas funções de coordenação do cuidado, pois a integralidade da assistência se torna fragmentada sem esse serviço.

Entretanto, o atendimento em UPAs tem demonstrado fragilidade no sistema de contrarreferência para a APS, o que acomete todo o sistema de saúde ${ }^{(16)}$. Ao considerar que o 'Protagonismo, Corresponsabilidade e Autonomia dos Sujeitos e Coletivos' está entre os princípios da $\mathrm{PNH}$, torna-se fundamental que trabalhadores e usuários reconheçam seu papel na produção de saúde ${ }^{(5)}$, promovendo a comunicação entre os pontos de atenção, como propõe o modelo de saúde brasileiro ${ }^{(15)}$.

\section{Dificuldades encontradas no processo de acolhimento nas UPAs}

Alguns percalços no processo de acolhimento nas UPAs também foram relatados pelos idosos e seus familiares. Dentre os quais sobressaem questões atreladas à procura indiscriminada nesses serviços que ocasionam demora no tempo de atendimento, problemas com a ambiência e recursos humanos e materiais incompatíveis ao número de usuários atendidos.

Exemplo mencionado foi o discurso de um idoso que procurou a Unidade para que fosse administrado medicamento prescrito por médico que acompanha seu problema de saúde, porém houve a necessidade em aguardar por consulta médica, para que o profissional transcrevesse sua medicação, mesmo em posse da receita médica: "Estou com anemia, a médica receitou seis medicações e tinha que aplicar intravenosa uma por semana, com soro. (...) Fui na UPA, mas, achei muito difícil a demora. Fui classificada com a cor azul e atenderam todo mundo na minha frente, só fui para tomar injeção e tive que passar pelo médico" (I21-Azul).

Embora haja registros de avanço do modelo assistencial brasileiro, para reorganizar a Rede de Atenção à Saúde (RAS) e potencializar sua intercomunicação-(13,16), observa-se que ainda há falhas na padronização de determinados procedimentos. Tal prática pode gerar desconforto entre os usuários, por não saberem qual serviço procurar, demonstrando a carência de informação referente às funções de cada ponto de atenção da RAS. Além de que, geram atendimentos desnecessários nas UPAs, os quais poderiam ocorrer diretamente na APS ${ }^{(3)}$. 
Contudo, há de se considerar que a interpretação equivocada das informações ou, até mesmo, exposição de informações limitadas, pode contribuir com demandas não urgentes nas UPAs $^{(4,13)}$. A superlotação dessas Unidades, acarreta em desprendimento de maior tempo no atendimento, o que provoca descontentamento ao usuário no processo de acolher, já que tal aspecto corresponde a uma virtude do acolhimento, bem como gera insatisfação nos profissionais, decorrente da falta de continuidade no trabalho(2).

Associada ao tempo de espera, também se constata fragilidade referente à disponibilização pelos resultados de exames. Vale destacar que este é um desafio enfrentado nos serviços de saúde, de modo geral, portanto corrobora com a literatura, porque o tempo para ser atendido e para receber o resultado dos exames representa motivo de alta insatisfação pela população ${ }^{(17)}$.

Para os participantes, deste estudo, acarreta em atraso na conduta da equipe médica sobre o desfecho da situação clínica do idoso: "Esperei muito tempo, porque tinha colhido um monte de exames, que demora de três horas e meia a quatro horas para ficar pronto. Quando ficou pronto, tive que esperar mais um tempão para o médico vir falar o que realmente tinha acontecido, se a minha mãe ia ficar internada ou se ia embora (...)" (F22-Vermelho). "O problema da UPA são os exames que tem que mandar para outro lugar e tem que ficar esperando um tempão pelo resultado. Não tem todos exames ali. Só colhe e manda para outro lugar, aí tem que esperar o resultado e depois resolve, se terá alta ou se precisará ficar internado" (I2-Verde).

Salienta-se que nenhuma das UPAs do município estudado possui em suas dependências laboratório para se fazer análise bioquímica dos exames. Pelo depoimento, nota-se que o usuário mencionou o encaminhamento do material biológico a outro serviço, localizado ao lado de uma das Unidades, responsável pela análise bioquímica dos exames dos serviços de saúde municipais $^{(8)}$.

Apesar do descontentamento do idoso, a legislação prevê laboratórios fora da Unidade, os quais possibilitam acesso a exames aos usuários com a existência de sala de coleta de materiais, dentro de intervalo de tempo tecnicamente aceitável e de acordo com parâmetros definidos pelas equipes locorregionais ${ }^{(3,11)}$. Reforça-se a importância em orientar a população sobre a organização de trabalho destes estabelecimentos de saúde, o que também corresponde a uma das formas de acolhimento.

Além do tempo de espera sobre os procedimentos realizados na Unidade, a infraestrutura também foi apontada como fragilidade no processo do acolhimento adequado. A sobrecarga, nesses serviços tem gerado superlotação, a qual torna a infraestrutura do serviço incompatível para atender a todos com qualidade: "Aquele ambiente de injeção, poderia ter mais cadeiras, mas ali é pequeno também, não dá. Eu acredito que seja por isso que demora para atender" (I21-Azul).

Associado ao número de cadeiras insuficientes, os participantes ressaltaram o desconforto proporcionado por estas: "Os mais novos estavam em pé nos corredores, porque não tinha cadeira para todos sentarem, e as cadeiras não eram confortáveis" (16-Verde). Vale destacar o relato de um idoso que estava de acompanhante e devido o desconforto das acomodações sofreu mal-estar e teve que ser atendido: "Naquele dia, eu fui na UPA porque eu acompanhava minha neta que estava com cólica de rim, aí como eu passei a noite inteira, sem dormir, sentada naquela cadeira horrorosa e dura, eu passei mal, aí fui atendida pelo médico" (E13-Amarelo).

Ainda referente à ambiência, os depoentes ressaltaram problemas com a higiene e conforto da UPA: "Talvez devido ter muita gente, não estava limpo, tem gente que joga as coisas no chão, uns acabam vomitando e outros pisam em cima. E também sobre a ventilação, nós pedimos para a enfermeira regular um pouco $o$ ar condicionado, porque estávamos com frio" (16Verde).

Sabe-se que a infraestrutura compromete a assistência de enfermagem para o cuidado à população idosa ${ }^{(18)}$. Isso também vai contra as diretrizes da $\mathrm{PNH}$, que preconiza que os estabelecimentos de saúde tenham estrutura física e ambiência adequadas, a fim de que os usuários obtenham atendimento, confortavelmente, amenizando seus sofrimentos ${ }^{(5)}$.

A ambiência é fortemente atrelada ao acolhimento. Na saúde, refere-se ao espaço físico compreendido como espaço social, profissional e de relações interpessoais que deve possibilitar atenção acolhedora, resolutiva e humana ${ }^{(19)}$. Para os participantes desta pesquisa, a ambiência das UPAs analisadas está aquém do esperado, principalmente, no que se refere ao conforto, limpeza e ventilação, limitando-se ao acolhimento 
conservador com práticas isoladas de triagem e organização de filas. Um estudo realizado em Atlanta-Geórgia corrobora essa condição, no qual o conforto das cadeiras esteve entre os fatores que mais influenciaram na insatisfação de idosos em serviços de emergência ${ }^{(19)}$.

Somada a essas questões, a superlotação nos serviços emergenciais não influencia apenas na sobrecarga da sua infraestrutura, produção de trabalho incompatíveis com o número de profissionais, o que para os idosos interfere diretamente no processo de acolhimento da UPA: "Quando fui atendida na UPA, tinham poucos profissionais, isso pode ter influenciado a demora, como na hora em que fui fazer o Raio-X, não havia nenhum funcionário, a sala estava fechada com um papel na porta informando que ele tinha ido na emergência infantil, aí ele demorou para fazer o meu Raio-X" (I6-Verde).

Segundo a PNH, os profissionais são elementos-chave para 0 sucesso do acolhimento $^{(5)}$, sendo que a sobrecarga de trabalho nas UPAs afeta os principais aspectos do acolhimento, como a escuta qualificada e a determinação de prioridades para atendimentos emergenciais ${ }^{(20)}$, além de implicar em maior tempo de espera para o atendimento. E, para àqueles que procuram os serviços em situações emergenciais, o tempo de espera para o atendimento representa a manutenção da vida.

\section{CONSIDERAÇÕES FINAIS}

Os dados obtidos neste estudo, possibilitaram conhecer como os usuários idosos e seus familiares compreendem o acolhimento em UPAs, os quais apontaram satisfação com o atendimento recebido pelos profissionais, principalmente relacionados à escuta qualificada e a resolutividade de problemas de saúde que motivaram a busca pelo serviço. Foram identificadas também algumas dificuldades enfrentadas na procura desse serviço caracterizados pelo tempo de espera, grande demanda, incompatibilidade da infraestrutura e a carência de profissionais.

Os resultados, deste estudo, pontuam aos gestores das UPAs a necessidade de implantar protocolos e estratégias que possibilitem melhorias na qualidade do atendimento, uma vez que a garantia de resolução das necessidades pode significar continuidade na qualidade de vida dessa população. Ainda aponta para a necessidade de reorganização dos serviços de saúde que compõem a RAS, sobretudo o fortalecimento e maior investimento na APS, para que exerça seu papel de coordenador e ordenador do cuidado, e os usuários utilizem, adequadamente, os serviços primários, de modo que a qualidade e organização das UPAs sejam impactadas positivamente. E, a importância dos profissionais de saúde no empoderamento da população para a procura consciente dos serviços de saúde emergenciais.

No que diz respeito ao envolvimento dos profissionais nas práticas de acolhimento, há de se considerar que a enfermagem tem no seu contexto a arte do cuidar e constitui a categoria com maior número de profissionais nas UPAs, portanto, julga primordial a consolidação dos dispositivos da $\mathrm{PNH}$, por esses membros da equipe, para que promovam a qualificação da assistência. Assim, espera-se que este estudo possibilite a reflexão desses profissionais sobre a assistência oferecida à população idosa nesses serviços.

Como limitação, o estudo traz exclusivamente a percepção dos idosos e familiares atendidos em UPAs, o que demonstra que novas pesquisas com os profissionais de saúde podem ser promissoras para o investimento em saúde e para o avanço do conhecimento em enfermagem e saúde. Entretanto, o impacto social dos resultados encontrados, visto que a qualidade do acolhimento reflete no tempo e no desfecho do cuidado ao idoso, traz contribuições à área da saúde e à enfermagem e poderá subsidiar novos estudos que visam a aprimorar o acolhimento de idosos nos serviços de saúde emergenciais.

\section{REFERÊNCIAS}

1- Zhao C, Wong L, Zhu Q , Yang H. Prevalence and correlates of chronic diseases in an elderly population: A community-based survey in Haikou. PLoS One 2018;13(6):e0199006. DOI: 10.1371/journal.pone.0199006

2- Schwarz UVT, Hasson $\mathrm{H}$, Athlin AM. Efficiency in the emergency department: A complex relationship between throughput rates and staff perceptions. Int Emerg Nurs. 2016;29:15-20. DOI: 10.1016/j.ienj.2016.07.003

3- Uchimura LYT, Viana ALD, Silva HP, Ibañez N. Unidades de Pronto Atendimento (UPAs): Características da gestão às redes de atenção no Paraná. Saúde Debate 2015;39(107):972-83. DOI: 10.1590/0103-110420151070253

4- Gonçalves AVF, Bierhals CCK, Paskulin LMG. Acolhimento com classificação de risco em serviço de emergência na perspectiva do idoso. Rev 
Gaúcha Enferm. 2015;36(3):14-20. DOI: 10.1590/1983-1447.2015.03.52422

5- Brasil, Ministério da Saúde. HumanizaSUS: Documento base para gestores e trabalhadores do SUS. 4a ed. Brasília, DF: Ministério da Saúde; 2010 6- Luiz FF, Caregnato RCA, Costa MR. Humanização na terapia intensiva: Percepção do familiar e do profissional de saúde. Rev Bras Enferm. 2017;70(5):1040-7. DOI: 10.1590/00347167-2016-0281

7- Ferreira BR, Silva FP, Rocha FCV, Oliveira ADS, Amorim FCM. Acolhimento ao idoso na atenção básica: Visão do usuário. J Res: Fundam Care Online 2018;10(3):669-74. DOI: 10.9789/21755361.2018.v10i3.669-674

8- Martins NIM, Caldas PR, Cabral ED, Lins CCSA, Coriolano MGWS. Instrumentos de avaliação cognitiva utilizados nos últimos cinco anos em idosos brasileiros. Ciênc Saúde Coletiva 2019;24(7):2513-30. DOI: 10.1590/141381232018247.20862017

9- Manzini EJ. Considerações sobre a elaboração de roteiro para entrevista semiestruturada: Colóquios sobre pesquisa em educação especial. Londrina: Eduel; 2003

10- Strauss A, Corbin J. Pesquisa qualitativa: Técnicas e procedimentos para 0 desenvolvimento de teoria fundamentada. $2 \mathrm{a}$ ed. Porto Alegre: Artmed; 2008

11- Inteligência Artificial do Paraná. Unidades de pronto atendimento: rede de urgência e emergência. Paraná: PIÁ; 2014

12- Hermida PMV, Nascimento ERP, EchevarríaGuanilo ME, Brüggemann OM, Malfussi LBH. User embracement with risk classification in an emergency care unit: An evaluative study. Rev Esc Enferm USP 2018; 52:e03318. DOI: 10.1590/s1980-220x2017001303318

13- Leite TEHP, Sousa FIN, Ponte VA, Mariano MR, Barbosa PME, Araújo TM. Os desafios da humanização dentro de unidades de pronto atendimento: A visão dos gestores. Cienc Cuid Saúde 2018;17(2):1-8. DOI: 10.4025/cienccuidsaude.v17i2.41866

14- Azevedo, E. Alimentação, sociedade e cultura: Temas contemporâneos. Sociologias 2017;19(44):276-307. DOI: 10.1590/15174522$\underline{019004412}$

15- Hermida PMV, Nascimento ERP, EchevarríaGuanilo MEI, Andrade SR, Ortiga ÂMB. Contrarreferência em Unidade de Pronto Atendimento: discurso do sujeito coletivo. Rev Bras Enferm. 2019;72(supl 1):143-50. DOI: 10.1590/0034-7167-2018-0023
16- Mendes EV. As redes de atenção à saúde. Ciênc Saúde Coletiva 2010;15(5):2297-305. DOI: 10.1590/S1413-81232010000500005

17- Pedraza DF, Nobre AMD, Menezes TN. Avaliação da estratégia saúde da família na perspectiva dos idosos em Campina Grande, Paraíba. Rev APS. 2018;21(1):37-47. DOI:

10.34019/1809-8363.2018.v21.16142

18- Boltz M, Parke B, Shuluk J, Capezuti E, Galvin JE. Care of the older adult in the emergency department: Nurses views of the pressing issues. Gerontologist 2013;53(3):441-53. DOI: 10.1093/geront/gnt004

19- Cetin-Sahin D, Ducharme F, McCusker J, Cossette F, Vadeboncoeur A, MAH R. Experiences of an emergency department visit among older adults and their families: Qualitative findings from a mixed-methods study. J Patient Exp. 2019;1-11. DOI: 10.1177/2374373519837238/ 20- lordache S, Elseviers M, Cock R, Van Rompaey B. Development and validation of an assessment tool for nursing workload in emergency departments. Int J Nurs Clin Pract. 2020;29(56):794-809. DOI: 10.1111/jocn.15106

Nota: Extraído da dissertação: "Perspectivas de idosos sobre acesso e acolhimento em unidades de pronto atendimento", Programa de Pós-Graduação em Enfermagem, Universidade Estadual de Maringá, 2016.

Recebido em: 09/04/2020

Aprovado em: 08/07/2020

Endereço de correspondência:

Giovana Aparecida de Souza Scolari

Avenida Colombo, 5.790, Zona 7, Maringá-PR.

E-mail: giscolari@hotmail.com 